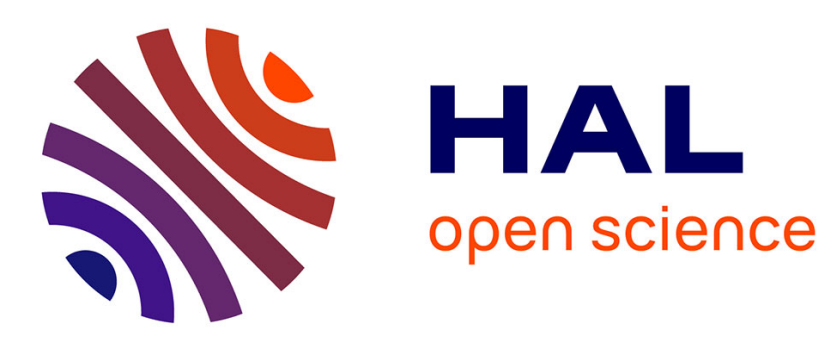

\title{
Mesure de la température de l'air avec l'abri cheminée type I.N.R.A
}

Rodrigo Antonioletti, André Pinguet, Jacques Saumade

\section{To cite this version:}

Rodrigo Antonioletti, André Pinguet, Jacques Saumade. Mesure de la température de l'air avec l'abri cheminée type I.N.R.A. Agronomie, 1987, 7 (9), pp.745-746. hal-00885050

\section{HAL Id: hal-00885050 https://hal.science/hal-00885050}

Submitted on 1 Jan 1987

HAL is a multi-disciplinary open access archive for the deposit and dissemination of scientific research documents, whether they are published or not. The documents may come from teaching and research institutions in France or abroad, or from public or private research centers.
L'archive ouverte pluridisciplinaire HAL, est destinée au dépôt et à la diffusion de documents scientifiques de niveau recherche, publiés ou non, émanant des établissements d'enseignement et de recherche français ou étrangers, des laboratoires publics ou privés. 


\section{Mesure de la température de l'air avec l'abri cheminée type I.N.R.A.}

Rodrigo ANTONIOLETTI, André PINGUET \& Jacques SAUMADE

I.N.R.A., Station de Bioclimatologie, Centre de Recherches d'Avignon, Stefce, F 84140 Montfavet

RÉSUMÉ

Un abri miniaturisé à coupelles, conçu pour la mesure de la température de l'air, a été comparé à un abri météorologique classique. La différence moyenne de température pour l'ensemble des observations est de $0,16{ }^{\circ} \mathrm{C}$, de même ordre pour les températures minimales, et de $0,3{ }^{\circ} \mathrm{C}$ pour les maximums.

Mots clés additionnels : Abri à coupelles, température de l'air, mesure de la température.

A small circular-vaned shelter for temperature measurements was compared with a standard meteorological screen. Observed mean difference between shelters was about $0.16^{\circ} \mathrm{C}$ for continuous and minimum temperature, and about $0.3{ }^{\circ} \mathrm{C}$ for maxima.

Additional key words : Tiled shelter, air temperature, temperature measurement.

\section{INTRODUCTION}

L'utilisation de capteurs de type numérique ou analogique devient de plus en plus courante dans la pratique des mesures météorologiques. Les avantages principaux en sont, d'une part, la précision, et, d'autre part, la possibilité de sauvegarder les informations collectées sur un support pouvant être traité directement à l'ordinateur.

Cette tendance à l'automatisation des mesures implique une évaluation des nouvelles conditions requises pour réaliser la mesure, vis-à-vis des influences radiatives, comme c'est le cas dans la mesure de la température de l'air. Ainsi, plusieurs types d'abris, à ventilation forcée et autoventilés, ont été comparés aux abris météorologiques classiques de type Anglais et plastique petit modèle (ANTONIOLETTI et al., 1983).

L'objet de cette note est de comparer la réponse d'un abri à coupelles de petite taille, réalisé au STEFCE (Station de Bioclimatologie d'Avignon), actuellement commercialisé par la Société Cimel et utilisé pour la mesure de la température de l'air dans les stations automatiques de l'I.N.R.A., à celle d'un abri météorologique classique.

L'encombrement de ce petit abri est de $200 \mathrm{~mm}$ de haut, dont $60 \mathrm{~mm}$ correspondent au support, et un diamètre maximal de $81 \mathrm{~mm}$. La hauteur de chaque coupelle est de $17 \mathrm{~mm}$, avec un écartement de $12 \mathrm{~mm}$ entre les coupelles (fig. 1). Le poids de cet abri est de $300 \mathrm{~g}$.

\section{COMPARAISON DES ABRIS}

L'essai a porté sur la mesure de la température de l'air dans un dispositif comprenant un abri cheminée modèle I.N.R.A., à ventilation naturelle, et un abri météorologique plastique petit modèle, de la Météorologie Nationale.

Pour la mesure de la température on a utilisé des sondes à résistance de cuivre, avec $\mathrm{R}=2330 \mathrm{ohms}$ à $0{ }^{\circ} \mathrm{C}$, qui fournissent une réponse linéaire de $10 \mathrm{ohms} /{ }^{\circ} \mathrm{C}$. Avant sa mise en expérimentation en plein air, on a contrôlé les sondes dans un bain d'éta- 


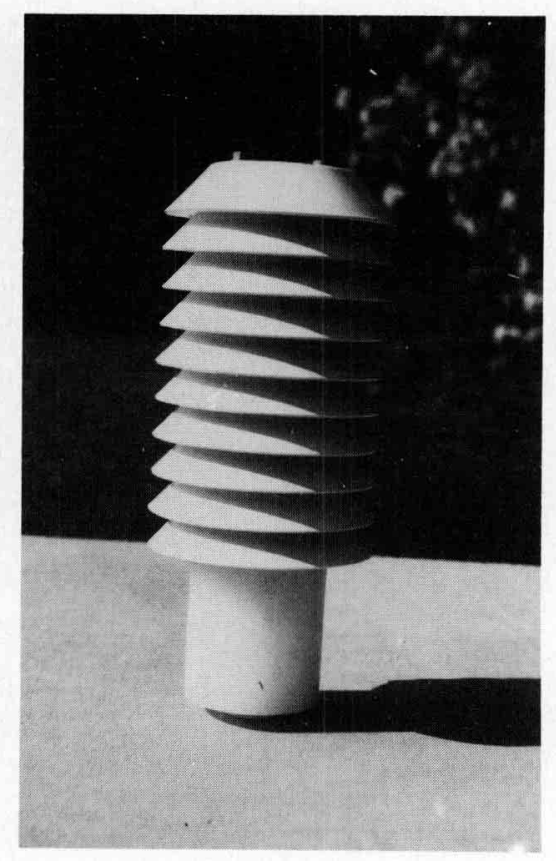

Figure 1

Abri cheminée utilisé dans le réseau de stations climatologiques automatiques de I'I.N.R.A.

Circular-vaned shelter in use for I.N.R.A. 's automatic climatological network.

lonnage à différentes températures. La précision de la mesure étant de $0,1^{\circ}$.

Une station automatique CIMEL assurait l'enregistrement des valeurs toutes les 6 minutes ; les données étaient stockées dans une mémoire EPROM et dépouillées ensuite à l'ordinateur. L'expérimentation s'est déroulée sur 95 jours, de juillet à octobre (ANTONiOletTI \& PingueT, 1985).

Pour l'ensemble des observations relevées toutes les 6 minutes, l'écart de température entre les 2 abris a varié entre $-0,3$ et $+0,8{ }^{\circ} \mathrm{C}$; cette variation est
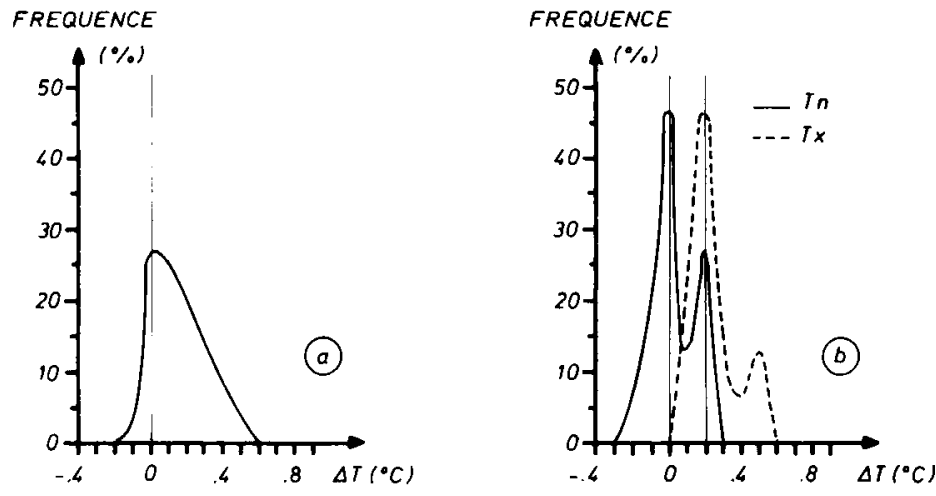

Figure 2

Fréquence des écarts de température entre les abris cheminée et plastique petit modele:

a) valeurs toutes les 6 minutes,

b) températures extrêmes.

Relative distribution curve of temperature differences between tiled and standard shelters :

a) for values every 6 minutes,

b) for maximum and minimum values.

liée, pour l'essentiel, à la différence d'inertie entre les enceintes, l'abri cheminée réagissant plus rapidement aux variations de température. La moyenne des écarts est de $0,16{ }^{\circ} \mathrm{C}$ (fig. 2a).

En ce qui concerne les températures extrêmes, valeurs les plus couramment relevées sur les documents climatologiques, la dispersion est moindre. On a une correspondance à $+0,2{ }^{\circ} \mathrm{C}$ pour $95 \mathrm{p} .100 \mathrm{des}$ températures minimales, et une légère surestimation des températures maximales, de 0,1 à $0,5^{\circ} \mathrm{C}$, pour 95 p. 100 des observations, la plus grande fréquence (46 p. 100) étant de $0,1{ }^{\circ} \mathrm{C}$ (fig. 2b). Dans les 2 cas, la courbe de distribution des écarts présente une dissymétrie positive, mais un peu plus accusée pour les maximums, ce qui est normal étant donné que l'influence des facteurs radiatifs est plus importante de jour que de nuit.

\section{RÉFÉRENCES BIBLIOGRAPHIQUES}

Antonioletti R., Pinguet A., Saumade J., 1983. Essais d'abris à ventilation statique ou dynamique pour la mesure de la température de l'air. Avignon, I.N.R.A.-STEFCE, note 83.8, 16 p.
Antonioletti R., Pinguet A., 1985. L'effet d'abri dans la mesure de la température de l'air. L'abri cheminée type I.N.R.A. comparé aux normes de la Météorologie Nationale. Avignon, I.N.R.A.-STEFCE, note 85.20 . 